\title{
CPAS Airdrop Test Joint Recertification by Analysis
}

\author{
Charles J. McCann ${ }^{1}$ \\ NASA Johnson Space Center, Houston, TX, 77058, USA \\ and \\ Fernando S. Galaviz ${ }^{2}$ and Satish Reddy ${ }^{3}$ \\ Jacobs Technology/JETS, Houston, TX, 77058, USA
}

\begin{abstract}
Airdrop testing of parachutes is a complicated endeavor that requires the custom design and certification of many critical components. The most direct path to certifying a component is to perform full scale testing with margin over the maximum loads expected to be seen in operation. However, other constraints often preclude the opportunity to perform full scale testing. In this paper, we present a case study where a problem arises in a joint that had been certified with a full scale test. There was no time or budget available to repeat the full scale testing after a redesign of the joint. Instead, we present a method of testing each failure mode at the component level to support a certification by analysis approach. The analysis itself was not complicated, but tradeoffs had to be made between different failure modes to arrive at the optimal design. The same approach was also applied back to the original joint to confirm that the failure mode that was not seen in full scale testing would have been caught by the proposed analysis. In the end, the new design was certified by analysis and worked without issue for the final six airdrop tests that used this joint.
\end{abstract}

\section{Nomenclature}

$C M \quad=$ center of mass

$C P A S=$ capsule parachute assembly system

MOS = margin of safety

$P C D T V=$ parachute compartment drop test vehicle

PHDJ = PTV Hold Down Joint

$P T V \quad=$ parachute test vehicle

$W L L \quad=$ working load limit

\section{Introduction}

$\mathrm{T}$ HE Capsule Parachute Assembly System (CPAS) Project pushed the state of the art not only for parachute design, but for the techniques used in airdrop testing as well. A number of safety critical components required a custom design. In this paper, we look at one such component that was originally certified with a full scale test, and then needed to be recertified by analysis after experiencing a failure.

First, we look at the CPAS airdrop testing configuration background to understand the purpose of this joint. We then describe the specific configuration of the joint in question, as well as the full scale testing that was performed to certify this joint for flight.

While the full scale testing and the first eleven airdrop tests using this joint configuration showed no indications of an issue. The joint experienced a failure while being configured for the first CPAS qualification test. Details are presented for the observed failure as well as the proposed change to the design.

The most involved section of the paper deals with the analysis that was done to recertify this joint for use in airdrop testing after the failure. Multiple tests were completed to understand the observed failure mode and to create corresponding knockdown factors for use in the analysis. At the same time, we had to confirm that making the joint

${ }^{1}$ Systems Engineering and Integration Lead, CPAS Project, Project Management and Integration Office (EA5).

${ }^{2}$ Simulations and Analysis Engineer, Engineering, 2224 Bay Area Boulevard, Houston, TX 77058.

${ }^{3}$ Chief Engineer, Engineering, Jacobs Bldg 1, 2224 Bay Area Boulevard, Houston, TX 77058. 
stronger did not do anything to adversely affect the function of the joint. The overall strategy for the analysis is presented, but not the specific values. These can be made available by request, but most likely it is the approach, and not the specific results that can be replicated on future projects.

\section{CPAS Airdrop Testing Configuration Background}

CPAS used Airdrop Testing as a critical component of system certification. For all airdrop tests, there was a parachute compartment that replicated the forward bay of the Orion crew module where all of the parachutes are attached. The parachute compartments could be mated to either a Parachute Test Vehicle (PTV) or a Parachute Compartment Drop Test Vehicle (PCDTV). The PTV was used to generate a representative wake, while the PCDTV was used to generate maximum dynamic pressure at deployment. In either case, one of the most complex aspects of the test technique was how to extract the test vehicle safely from the back of the aircraft.

For the PTV, extraction was completed by attaching it to a platform assembly called the Cradle and Platform Separation System (CPSS) as shown in Fig. 1. The CPSS attached to the rail system inside the carrier aircraft to control the motion of the test article from the position where it is secured to the exit point. Tight control of this motion is required for the PTV because it was designed to use the full extent of the size envelope for extracting payloads. A full Orion crew module would not fit out the door, so the PTV height was decreased just enough to get through.

While the CPSS to aircraft interface was a standard connection, the connection between the PTV and the CPSS was a custom design. The two driving requirements for this joint were to hold the PTV tightly to the CPSS while inside the
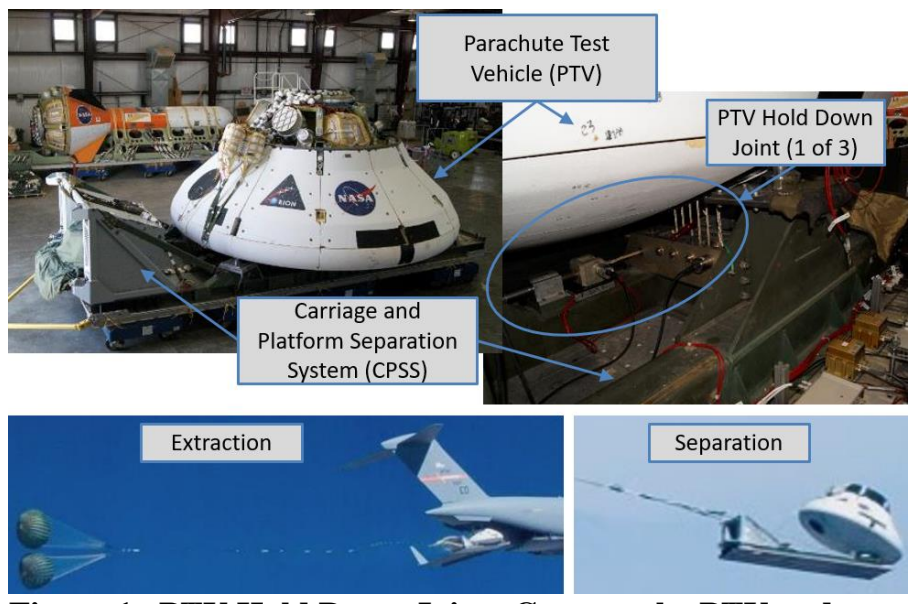

Figure 1. PTV Hold Down Joints Connect the PTV and CPSS While on the Aircraft and Through Separation.

aircraft for the same reasons described above, while being easy to separate just after extraction to allow the test vehicle to drop independently from the CPSS. The configuration and performance of this PTV Hold Down Joint (PHDJ) is the subject of this paper.

\section{Joint Configuration}

The connection of the PTV to the CPSS is made with three PHDJs as shown in Fig. 2. They are not distributed with an even 120 degree spacing around the vehicle because the CPSS is not as wide as the full diameter of the PTV. Instead, there are two joints that are closer together towards the rear of the aircraft, labeled "Port" and "Stbd," and one joint in the middle of the CPSS towards the front of the aircraft, labeled "Front."

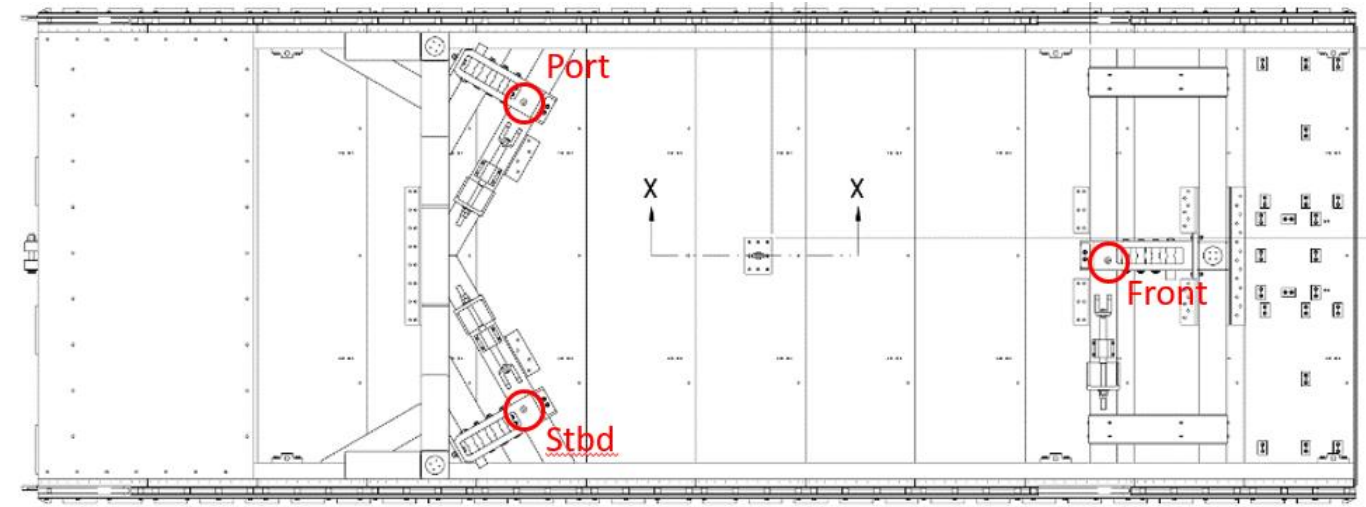

Figure 2. Location of Three PHDJs on CPSS. 
Each PHDJ consists of a ball and socket connection that is preloaded by a Vectran cord as shown in Fig. 3. The ball and socket positions the PTV correctly on the CPSS, helps prevent lateral motion, and allows for a clean release during separation. The Vectran cord constrains the PTV in the vertical direction and provides a simple means for separation as off the shelf cutters are used to cut the cord after extraction.

The Vectran passes over a series of pulleys to increase the amount of force that can be applied in the vertical direction. There are three pulleys inside the

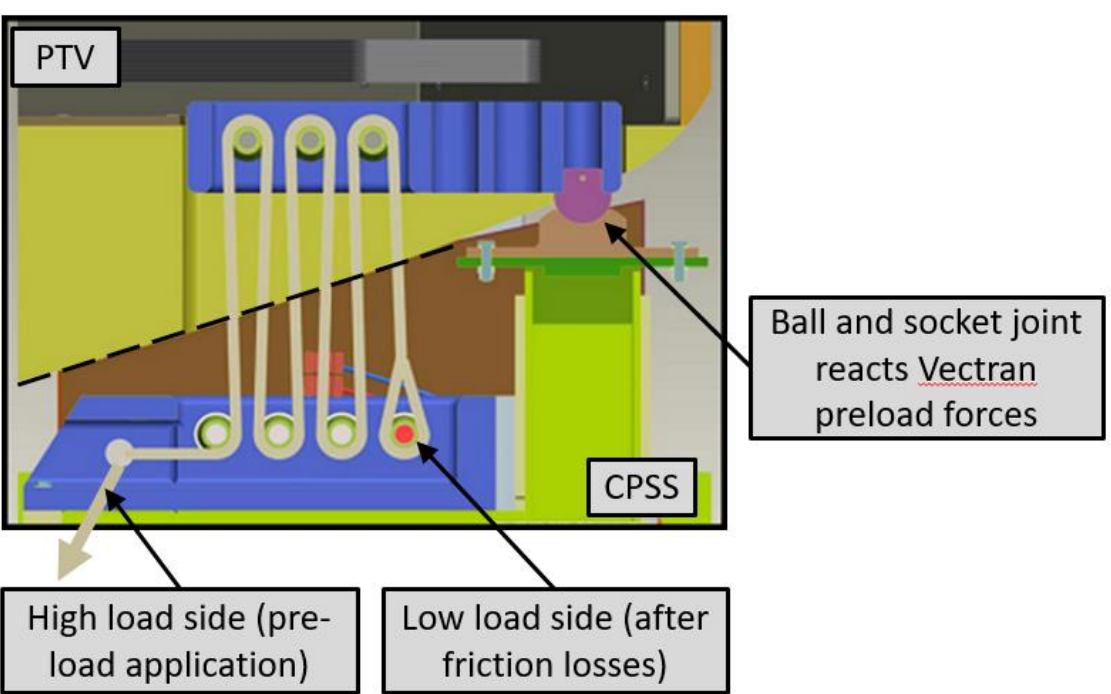

Figure 3. PTV Hold Down Joint Configuration.

outer mold line of the PTV and four pulleys located on the CPSS. Passing the Vectran over these pulleys works much like a block and tackle hoist. A single input tension is multiplied by six to match the number of times the Vectran is routed between the PTV and CPSS, while subtracting the friction losses that occur at each pulley.

The Vectran is connected to the CPSS by creating a loop on each end that passes over a pin. The loops are created with a Brummel knot. One of the CPSS pins is stationary, while the other is attached to a screw jack that is rotated to apply tension to the joint. Each tensioning pin on the CPSS has a load cell that was used to measure the amount of tension input to the joint.

To understand the friction losses over each joint a test stand was created to represent the pulley configuration and tensioning system as shown in Fig. 4. All of the pins on the test stand were instrumented, so that the total force could be measured directly. As expected, the force decreased for pins that were further away from the input tension. However, repeated applications of the input tension, with time in-between to allow the system to settle, reduced the losses observed throughout the system. Following this process not only minimized the losses, but made them consistent enough that the total preload could be accurately estimated by measuring only the loads on the first and last pins.

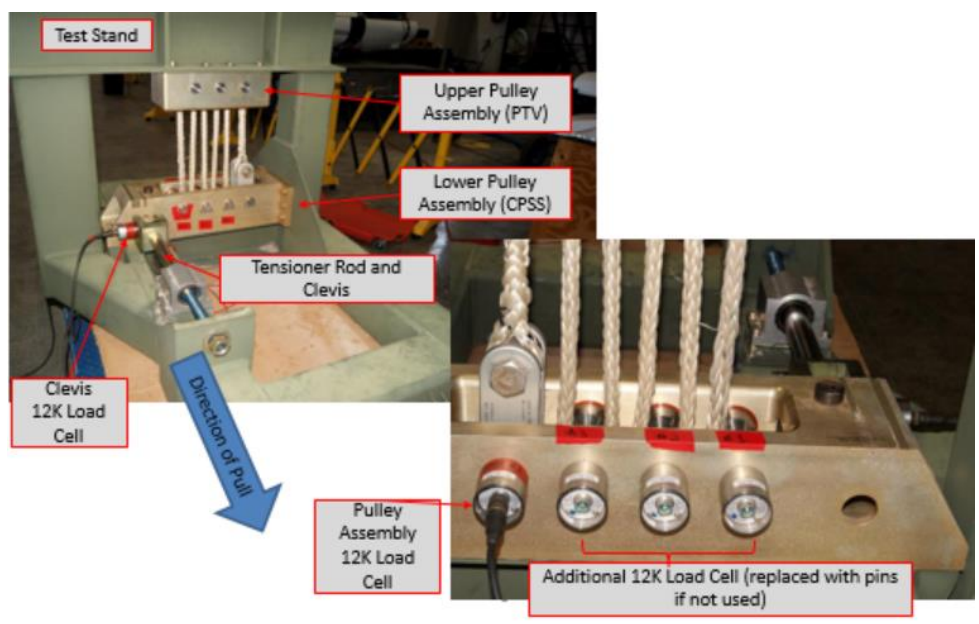

Figure 4. Test Stand Representation of PHDJ

\section{Original Joint Certification by Test}

Considering the criticality of this joint, the original certification that it would work properly was done with full scale static load testing. Calculations had been made to size the joint, but the test was performed to prove it out.

The full scale testing consisted of two primary configurations. For testing in the forward, aft, and lateral directions, the CPSS was hard mounted, and the static equivalent of the acceleration load requirements were applied to the PTV. Linear Variable Differential Transformers measured the deflection at each PHDJ, and the preload before and after the test was also monitored. A compression load test was completed by stacking weights on the PTV with similar measurements. No testing in the upward direction was completed. All of these tests showed acceptable deflections, consistent preload, and no failed hardware. The PHDJs were certified for use during airdrop tests based 
on these tests, and 33 total preloaded joints worked perfectly during the 11 PTV tests performed during the development test series.

\section{Failure Description}

After the development test series, the design of the parachutes had been baselined at the Critical Design Review, and the CPAS team believed most of the test technique issues had been worked out. It was now time to repeat what had been done before with flight-like parachutes in a series of eight qualification tests, six of which, including the first one, were to be configured with a PTV.

As the team went through the normal PTV build up test procedures, it came time to tension the PHDJs. The three locations were tensioned, but when we came back to check them, the Front PHDJ had lost most of the input preload. This was unusual, but could happen if something had slipped. Tension was reapplied and the Vectran cord snapped right at the end of the Brummel knot tail on the high load side of the cord as shown in Fig. 5. The qualification test series was derailed before it even started. However, the team was able to shift plans rapidly to build up a PCDTV for the first qualification test and create some time to investigate this failure.
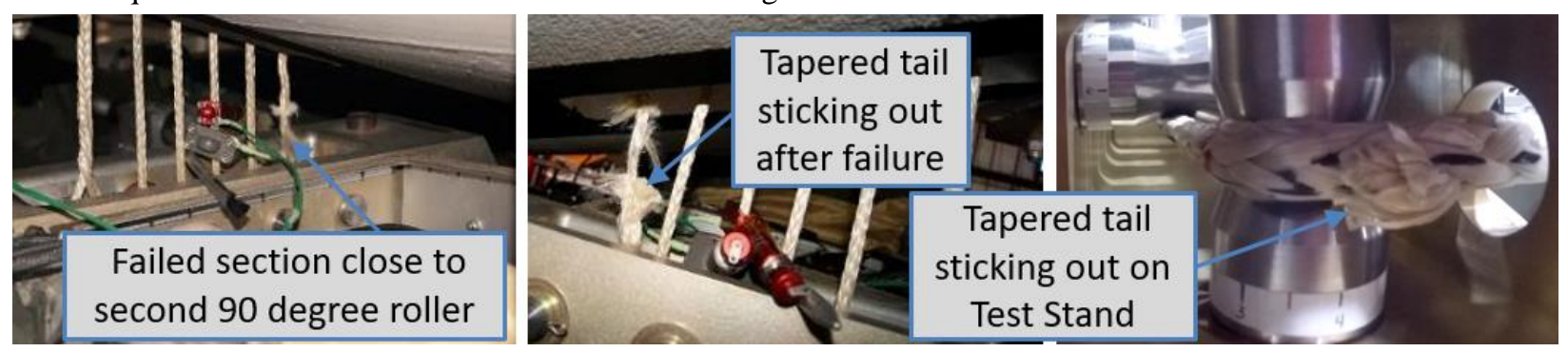

Figure 5. Failure Observed on CQT 4-2.

The first part of the investigation was to take a new Vectran cord and try to preload the joint in the test stand with as much video coverage as possible. This resulted in an interesting finding. When tension is first applied to the Vectran cord, there is a lot of stretch and a lot of slack that is pulled through the system. As the cord moves, there comes a point where the end of the inserted tail that was used to create the Brummel knot passes over a pulley. The video showed that as this tail was loaded over the curvature of the pulley it would cause the internal fibers to stick out through the outer braid of the Vectran. This would create a stress concentration that had not been used in the sizing analysis and may not have been present during the full scale tests. The cord on which this phenomena was observed did not fail, but it was still determined to be a credible failure mode. Looking at the failed ends of the cord that broke on the PTV under a microscope showed they were curved, which would indicate that the failure location was wrapped over a pulley at the time of failure.

A second part of the early investigation was to test the smaller Vectran cord with the Brummel tail wrapped over a pulley. This showed that the failure could be repeated, and the knockdowns observed were higher than expected. The test derived knockdowns showed that the smaller diameter Vectran could not be certified by analysis, even though it had passed the full scale tests.

With the scheduled date for the second qualification test fast approaching, the team decided a design change was needed for the PHDJs based on these initial investigations.

\section{Design Changes}

The primary design change was to increase the diameter of the Vectran cord. This provided a few advantages. The overall strength capability of the cord more than doubled. The modulus of the cord also increased such that we could place the ends of the inserted tails in a position that, even after moving the maximum amount while tensioning, they still would not pass over a pulley to create a stress concentration similar to what was caught on video with the smaller diameter cord.

Another advantage was that the rest of the joint had originally been designed to be used with the larger cord. Therefore, the curvature of the pulleys was already optimized to reduce the knockdown factor for that cord, and cutters that could sever this cord had already been identified and tested.

There were also some tradeoffs for going to the larger diameter cord. It did not slide over the pulleys as easily, so even though a higher input tension could be generated, there were also much greater losses in the system. This was the main reason that the original design of the joint did not use the lager cord. By using the test stand, we determined that even with a higher input tension the total preload on the joint was lower when compared to the smaller cord. 
A final, and perhaps most important, drawback of using the larger diameter cord was that it would invalidate the full scale testing that had been done to certify this joint. For the first two issues, we believed that the significant increase in both ultimate strength and modulus would more than cover for these drawbacks. However, invalidating the full scale testing with no time or budget available to repeat it, left us in a difficult position. We could not stick with the current design having just observed a failure that could threaten not only successfully completing a test, but the safety of the crew onboard the aircraft. Therefore, we decided to pursue the design change with a plan to recertify the joint by analysis, supported by a series of component level tests to determine joint knockdown factors.

\section{New Approach to Joint Certification by Analysis}

We had to address two primary concerns to certify the new PHDJ design by analysis. The first concern was the tensile strength of the Vectran considering all of the associated knockdown factors for the configuration in which it was being loaded. This analysis included a check for creep rupture and was meant to show we would not repeat the failure observed with the smaller diameter cord. The second primary concern was to evaluate the effectiveness of the joint to prevent gapping and sliding at the ball and socket joint with a lower total preload.

A difficulty in the analysis was that a higher applied preload would reduce margins for the strength of the Vectran, while improving the effectiveness of the joint. Therefore, the final preload value selected would need to be a balance between the two. When considering this balance, we had to take into account the consequence of each failure mode. As soon as the Vectran breaks, the PTV becomes unrestrained and presents a safety risk to the aircraft and crew. On the other hand, calculations for gapping and sliding identified the initiation of movement in the ball and socket joints. If the PTV started to move, but the acceleration load was not sustained, the PTV would likely drop back into place. If the load was sustained, then the Vectran would pick up more load and could eventually fail.

Since the consequences of failing the Vectran directly in tension were worse than the consequences for some relative motion within the ball and socket joints, we decided to bias the allowable preload towards not failing the Vectran. To do this, we used a higher factor of safety for the Vectran tensile failure and made conservative assumptions within this analysis.

The approach to calculating margins against a tensile failure of the Vectran was to empirically determine the knockdown factors. The tests would characterize how the larger Vectran cord managed the load in unique test configurations that are similar to the CPAS integration technique over rollers. The analysis objective was to compute a working load limit (WLL) and margin of safety (MOS) for the larger Vectran cord using test derived knockdowns at four designated locations for the Vectran cord in the PHDJs, as shown in Fig. 6. In addition, creep rupture concerns were addressed by demonstrating that operating loads are less than $50 \%$ of the breaking strength after knockdowns are applied.

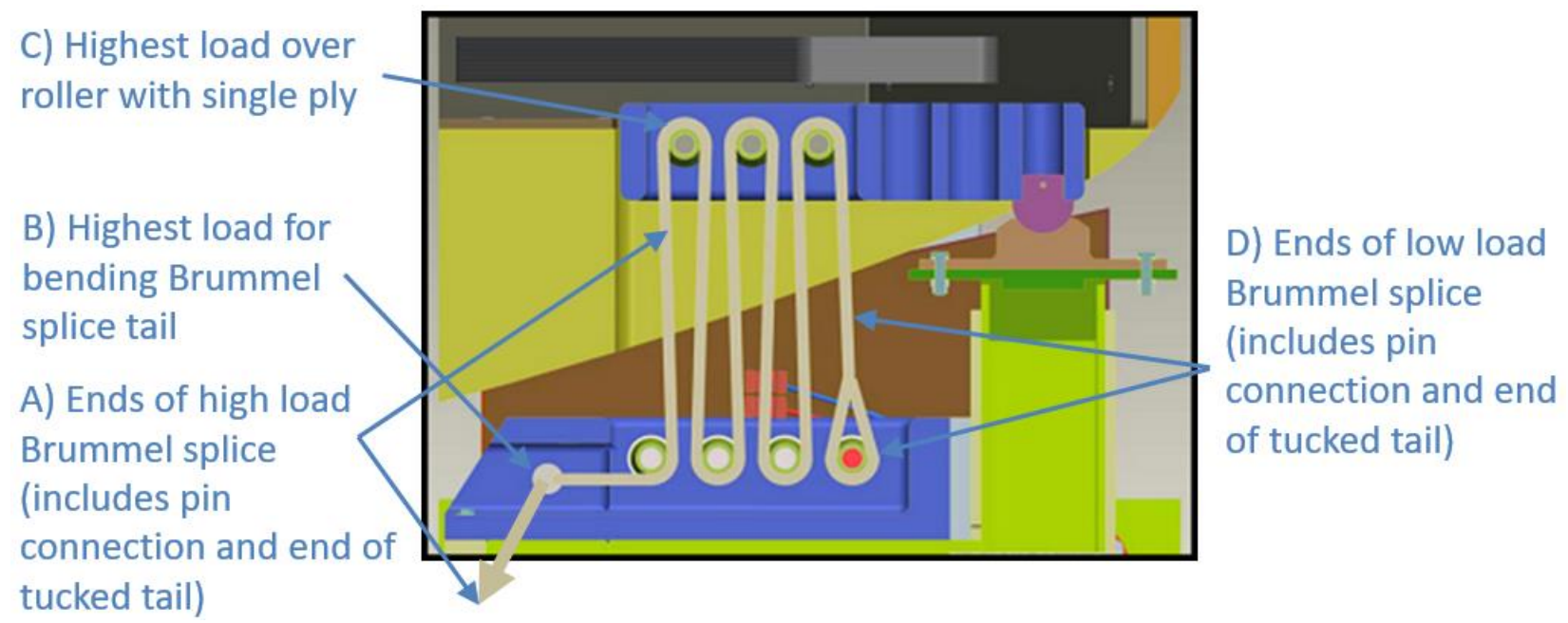

Figure 6. Locations of Test Derived Knockdown Factors.

A series of four tests, each containing six samples, were pulled to failure. These tests would establish the associated knockdowns to the material strength when configured into the testing apparatus, which was directly analogous to the CPAS integration technique in the PHDJ.

Test 1 was a straight pull to establish the splice knockdowns for the long and short Brummel tails, locations A and D, respectively in Fig. 6. A longer tail is used on the input side because of the higher loads. This knockdown essentially represents how the material strength is affected by the splice alone. 
Test 2 was a 90 degree pull to establish the knockdown for a quarter turn of the Brummel tail inside the base material. The long 22" tail Brummel was routed around a 90 degree bend, similar to location B, to demonstrate the material strength loss in this configuration. All test failures occurred at the end of the Brummel as expected.

Test 3 was the 180 degree pull to establish the half turn knockdown without a Brummel tail. The Brummel tail ends after the 90 degree roller and prior to the first 180 degree roller to ensure the end of a splice does not terminate around a pin, and therefore did not need to be checked over a 180 degree bend. The trailing section after the splice is considered the single ply portion and when routed over the 180 degree roller is subjected to the highest load, as seen in location C. All test failures occurred at the end of the Brummel as expected.

Test 4 was the flight-like integration knockdown to determine how subjecting the samples to multiple preload cycles similar to a flight integration influences the material strength of the Vectran. The test apparatus to determine the knockdown utilized the same 180 degree roller technique as Test 3; however, the samples were previously loaded and conditioned on the test stand. The purpose of this flight-like integration knockdown was to catch any unforeseen failure modes that were not captured with the other series of test.

For each test type, six cord samples were pulled to failure and the sample average, standard deviation, and applied knockdowns were calculated from the recorded test data. The knockdowns were calculated by simply taking the six sample average minus one standard deviation and dividing by the material lot strength.

Results showed that the worst case knockdown was for the 180 degree bend at location C. The magnitude of this knockdown was great enough to overcome the fact that the tension in the cord has decreased by the time it gets to this pin. Therefore, the input tension was selected such that it would create zero margin at this location, but with the high factor of safety and conservative assumptions.

Also for each location, the operating loads for the selected input tension were shown to be less than $50 \%$ of breaking strength calculated by taking the max expected applied load and dividing by the minimum breaking strength less one standard deviation. This eliminated concerns about creep rupture. To build even more confidence, a sample of the larger Vectran was installed on the test stand with full preload and allowed to sit for months to check for any long duration effects. None were observed.

The expectation based on test stand trials was that the input tension used to keep positive margin against a Vectran tensile failure would result in a total preload that was just over $8 \%$ lower than what had been applied with the smaller diameter Vectran. This raised the importance of being able to show by analysis that the joint would still prevent motion within the ball and socket joints.

All of the loads acting on the PTV were defined as acceleration loads applied to the center of mass (CM). This load was distributed to the three PHDJs using a finite element model. A linear analysis was assumed, so the preload from the Vectran tension was simply added to the results of the acceleration load reaction forces to get a total load at each PHDJ.

To calculate a margin for sliding, a mathematical description of the conditions for sliding had to be determined. For the PHDJs, the contact point between the ball on the PTV and the socket on the CPSS is 60 degrees from the centerline as shown in Fig. 7. The resultant force at each ball and socket joint can be calculated as a vector to determine the direction of the resultant force. As long as the direction of this force is inside of the 60 degree cone, it will be reacted directly by the socket. A resultant force outside of this 60 degree cone was used to define a sliding condition. In that case, the ball would start to slide up the socket, the load in the Vectran would increase, and hopefully static equilibrium would be reached before breaking the Vectran. To avoid the complexities of trying to analyze this case, we simply considered the initiation of sliding to be a system failure.

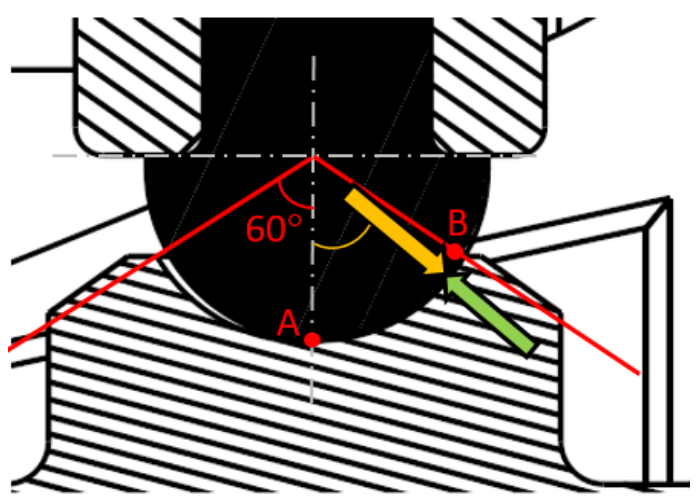

Figure 7. Conditions for Sliding.

To calculate a margin for gapping, the vertical acceleration that would need to be applied to the CM to create zero load in the vertical direction at any of the PHDJs was divided by the acceleration in this direction that could be applied to the test article.

\section{Results}

Using the expected total input torque and the load cases defined by MIL-STD-1791, we were able to show positive margin for both cases. Margins for gapping were lower than for sliding, and each decreased linearly as the total preload was lowered as shown in Fig. 8. 
This relationship became critical during the first use of the new PHDJ design. During the buildup for this test some anomalies occurred that resulted in a lower than expected total preload. However, with this graph, the margin of safety at any total preload force could be easily determined. For the total preload on CQT 4-2, we maintained positive margins by analysis, but trust in this analysis came under increased scrutiny because we were moving even further away from the preload that had been verified with full scale testing. In the end, the decision was made to fly CQT 4-2 with the lower preload value, and no anomalies were observed. For all future airdrop tests with the PTV, we achieved total preloads that were higher than CQT 4-2, but still not as high as what had been applied with the previous design. No anomalies with the PHDJs were observed for any of these test flights.

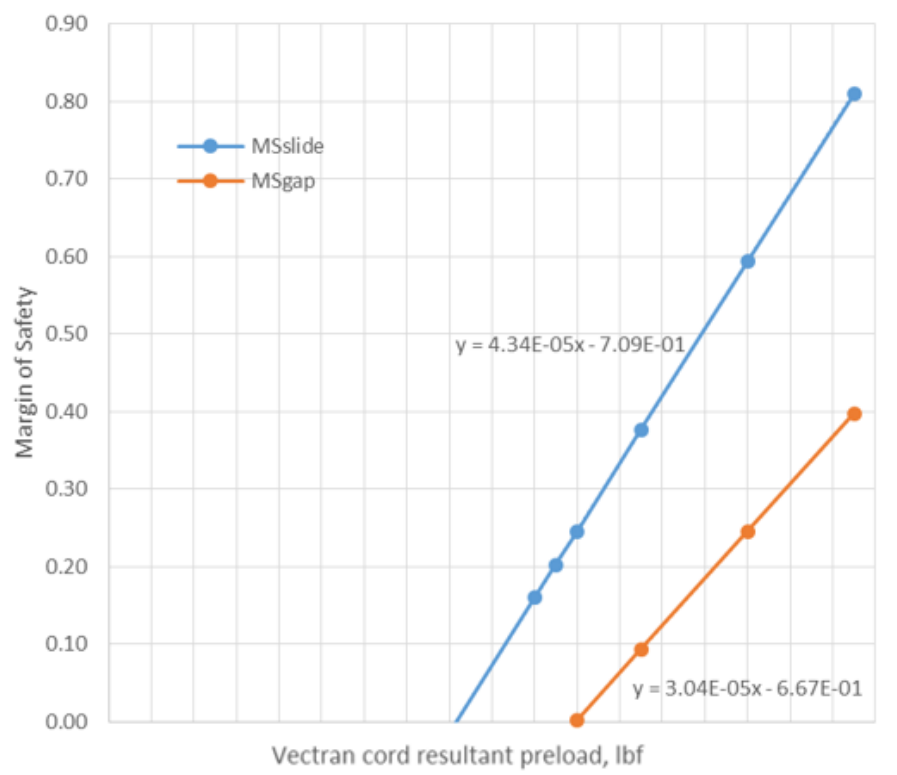

Figure 8. Effect of Preload on Margins for Sliding and Gapping.

\section{Conclusion}

CPAS qualification testing was the culmination of many years developing not only the parachutes, but also the test technique. As is often the case when testing hardware, there were some surprises found even when repeating what had been done before. In our case, the surprise resulted in the need to redesign a critical joint with important project schedules and budgets on the line. To do this, we replaced a full scale test with many smaller tests and analysis to interpret the results. The end result was a better joint design and a successful qualification test series. 